\title{
Diesseits und jenseits der sanften Apokalypse
}

Ich möchte noch einmal auf Maurice Blanchots Buch Le livre à venir zurückkommen, das übrigens in der deutschen Übersetzung den Titel Der Gesang der Sirenen trägt. Es führt uns in Blanchots Heimatland in die Zeit nach der „Libération“, in der Frankreich mit größter Energie versuchte, seine Rolle als „exception culturelle“ wiederzugewinnen, kulturpolitische und vor allem literarisch-philosophische Bemühungen, die im Übrigen bis in das Jahr 1980 erfolgreich waren.

So war die Zeit nach der Befreiung von der Occupation und dem Sieg über Nazideutschland nicht nur eine Epoche ausgelassener Freude über die wiedergewonnene Freiheit, sondern auch eine Zeit des Aufbruchs und des Suchens nach neuen Wegen. Dies galt auch für einen jungen Mann namens Roland Barthes, der in der Zeit nach dem Krieg, den er, an Tuberkulose erkrankt, vor allem in verschiedensten Sanatorien verbracht hatte, mühsam versuchte, sich als Lektor und mit allerlei kurzfristigen Anstellungen über Wasser zu halten. Sein Erstlingswerk Le degré zéro de l'écriture war 1953, kurze Zeit also vor Blanchots Buch, erschienen und markierte eine Schreibweise des Nullpunkts, die nicht mit der damaligen bundesdeutschen „Literatur der Stunde Null“ verwechselt werden darf.

Es ist für eine Analyse dieser Epoche aufschlussreich, dass sich Maurice Blanchot, der seit einigen Jahrzehnten - zumindest in bestimmten Theoriekreisen Frankreichs (und auch darüber hinaus) - wieder in Mode gekommen ist und für den man sich als großen Intellektuellen mit Gespür für die Entwicklungen seiner Zeit wieder zu interessieren scheint, in Le livre à venir bereits für Barthes' kleinen Band interessierte. Blanchot bezeichnete im letzten Teil seines 1959 erstmals veröffentlichten Buches unter dem Titel „Wohin geht die Literatur“ Roland Barthes' Le Degré zéro de l'écriture als „eines jener seltenen Bücher, in denen die Zukunft der Literatur sich abzeichnet". ${ }^{1}$

Vor diesem Hintergrund mag es nicht verwundern, dass sich gegen Ende von Blanchots Buch eine Zukunftsperspektive abzeichnete, welche von einem gegenwärtigen Zustand der Verwirrung gekennzeichnet sei, einer Verwirrung, wie sie Roland Barthes achtzehn Jahre später literarhistorisch rückblickend in seiner Leçon, seiner berühmten Antrittsvorlesung am Collège de France, festhalten sollte. Schauen wir uns die Schlusspassage dieses letzten Teils von Blanchots umfangreichem Band einmal näher an! Dabei möchte ich Ihnen eine Übersetzung ins Deutsche aus dem Gesang der Sirenen anbieten, die Sie bitte mit dem

1 Blanchot, Maurice: Der Gesang der Sirenen. München: C. Hanser, S. 279.

Ә Open Access. (C) 2021 Ottmar Ette, publiziert von De Gruyter. (cc) BY-NC-ND Dieses Werk ist lizensiert unter einer Creative Commons Namensnennung - Nicht-kommerziell - Keine Bearbeitung 4.0 International Lizenz. https://doi.org/10.1515/9783110703450-022 
Original vergleichen und als Beispiel für so viele Missverständnisse im deutschfranzösischen Theoriedialog verstehen mögen:

\begin{abstract}
Zu einer derartigen Verwechslung kommt es nicht zufällig. Das außergewöhnliche Durcheinander, dem es zu danken ist, dass der Schriftsteller veröffentlicht, ehe er schreibt, dass das Publikum formt und weitergibt, was es nicht versteht, dass der Kritiker beurteilt und definiert, was er nicht liest, dass schließlich der Leser lesen muss, was noch gar nicht geschrieben ist - diese Bewegung, die jedes Mal vorwegnehmend alle die verschiedenen Bildungsphasen eines Werkes zusammenwirft, versammelt diese auch in der Suche nach einer neuen Einheit. Daher die Fülle und das Elend, der Stolz und die Demut, die äußerste Verbreitung und die extreme Einsamkeit unseres literarischen Schaffens, dem wenigstens das Verdienst zuzusprechen ist, dass es weder nach Kraft noch nach Herrlichkeit verlangt. ${ }^{2}$
\end{abstract}

Die Einsicht von Maurice Blanchot, dass literarisches Schaffen weder an Macht noch an persönlichem Ruhm ausgerichtet sein dürfe, sollte eine ganze Reihe nicht allein französischer Denker der zweiten Hälfte des 20. Jahrhunderts prägen. Es ist, so sei hier eingefügt, für unsere Überlegungen nicht ganz nebensächlich, dass nach dem Zweiten Weltkrieg und der Libération jene Referenzbände oder diskursbegründenden Bücher gewechselt haben, die nach Ende der deutschen Besetzung die Diskussionen im Bereich von Ästhetik und Literatur prägten. Für lange Zeit war es Sartres berühmte Frage, Was denn Literatur sei, und mit ihr sein 1948 erstmals veröffentlichtes Buch Qu'est-ce que la littérature, das die Diskussionen weitgehend bestimmte und die Frage des politischen Engagements des Schriftstellers zentral stellte. Längst entwickelten sich hierzu alternative Konzepte und Verständnisweisen von Literatur und Gesellschaft sowie der Verbindungen zwischen beiden; doch blieben diese in den ersten Jahrzehnten nach dem Krieg im Hintergrund der spektakulären Debatten um den „écrivain engagé“ und entfalteten sich zunächst nur als einflussreiche Unterströmung.

Ein halbes Jahrhundert später wurde Sartres Literaturentwurf in Qu'est-ce que la littérature? überwiegend nur noch mit Blick auf seine Fragen nach dem aktiven Leser diskutiert, nach aktiven Formen der Rezeption und in den Kontext einer Fragestellung einbezogen, die bereits in Maurice Blanchots Le livre à venir aufgeworfen worden war: derjenigen nach dem Tod des Autors. ${ }^{3}$ Es mag sie überraschen, aber dies war in der Tat eine der zentralen Fragen in den Theoriedebatten der zweiten Hälfte des vergangenen Jahrhunderts. Die Wiederentdeckung Blanchots

2 Blanchot, Maurice: Le livre à venir. Paris: Gallimard 1959, S. 340.

3 Vgl. das Kapitel „Tod des letzten Schriftstellers“ in Blanchots Der Gesang der Sirenen, S. 295302. 
und das lange „Vergessen“ Sartres, dessen „purgatoire“ oder „Fegefeuer“ auch vier Jahrzehnten nach seinem Tod noch immer nicht beendet ist, bilden ebenfalls wichtige Hinweise auf grundlegende Veränderungen im literarischen und philosophischen Feld, die weit über Frankreichs Grenzen hinaus abstrahlten.

Dies alles waren wichtige Veränderungen und Umbesetzungen, welche auf der Ebene diskursiver Entwicklungen seit den sechziger Jahren und damit dem Ende der Hegemonie Sartres im literarischen und philosophischen Feld zusammen mit der neu erwachten Orientierung, dem neuen Interesse an den frühen Schriften Maurice Blanchots jene grundsätzliche Neuausrichtung einläuteten, die sich zwischen Moderne und Postmoderne situierte. Zuvor hatte Blanchot als ein Denker und Schriftsteller gegolten, der im Gegensatz zu Sartre nicht dazu in der Lage gewesen sei, Systeme zu bilden und systembildend zu wirken. Doch so schnell verändern sich die Zeiten. Derlei Qualitäten, wie sie Sartre zugesprochen worden waren, werden in Zeiten und im Zeichen der Postmoderne nicht mehr allzu sehr goutiert.

Was in der soeben angeführten Passage Blanchots dargestellt wurde, war nicht nur eine Konfusion, eine Verwirrung, sondern eine Deregulierung aller Mechanismen des Literaturbetriebs. Und zugleich - und für unsere Fragestellung vor allem - der Ausblick auf eine Literatur, die weder nach (politischer) Macht noch nach (literarischem) Ruhm strebe. Für beides hatten Philosophie wie Literatur Jean-Paul Sartres durchaus gestanden. Damit waren wesentliche Punkte einer Diskussion benannt, die sich - sicherlich nicht nur in Zeiten der Postmoderne zum einen um die Frage der Macht drehte, also jener Verbindung von Schriftsteller beziehungsweise Intellektuellem und „pouvoir“, dem Roland Barthes in seiner Antrittsvorlesung am Collège de France eine klare Absage erteilte. Denn für ihn war dieses Collège als der höchste und prestigeträchtigste Ort von Wissenschaft im damaligen Frankreich gerade jener Raum, der sich für ihn wunderbarerweise ,außerhalb der Macht' befand.

Der zweite Bereich war jener des Ruhmes, der „gloire“, also jenes hohe Prestige des „Großen Schriftstellers“, des „Grand écrivain“, an dessen Figur sich die Nouvelle Critique zu stören und ebenso zu reiben begann wie an der Kategorie des Werkes, des „Euvre“ und zumal des „Chef-d'œuvre“, des Meisterwerks. Begriffe und Kategorien, welche ein Barthes zusammen mit der Kategorie des Autors der Vergangenheit überantwortete. Es sollte ein für alle Mal Schluss sein mit den großen Schriftstellern und ihrem buchstäblich erlesenen Prestige und unvergänglichen Nachruhm. Es ist bei unserem kurzgefassten Rückblick sehr spannend zu sehen, wie die Frage nach dem Tod des Schriftstellers bei Maurice Blanchot, gewiss mit einer Menge an zeitgenössischem Theoriehintergrund, geradezu hausbacken daherkam, aber spätestens seit den ausgehenden sechziger Jahren erheblich an Fahrt aufnahm. In der vierten Phase beschleunigter Globalisierung und 
folglich im Kontext neuer elektronischer Medien und eines global vernetzten und ausgeweiteten literarischen Marktes mussten derlei Fragestellungen jedoch eine Brisanz erhalten, die man ihnen zu Beginn der Diskussion keineswegs zugetraut hätte.

Die Rede vom Ende der Literatur, welche keineswegs $\mathrm{zu}$ verwechseln ist mit der Rede vom Ende des Romans, öffnete sich rasch zu einer Rede vom Ende ihres Subjekts, des Autors und mehr noch des Subjekts aller Subjektphilosophie, des Menschen selbst. Denn immer deutlicher wurde herausgestellt, dass der Mensch keine Essenz, sondern eine Konstruktion sei, die in einer bestimmten Kultur und keineswegs in allen Kulturen - zu einem bestimmten Zeitpunkt erfunden wurde und daher auch ein Ende, eine Art Verfallsdatum haben müsste. Schnell also weiteten sich in der zeitgenössischen Philosophie die „enjeux“ und die Kernfragen aus, befeuert aber - und darauf lege ich größten Wert - von den entscheidenden Anregungen und Ausformungen im Bereich der Fiktion, im Bereich der entstehenden Literaturen der Welt, welche ein Denken formulierten, das mit einer deutlichen zeitlichen Verzögerung in der, westlichen' Philosophie ankam. Auf diese Pionierstellung der Literaturen der Welt werden wir im weiteren Verlauf unserer Vorlesung immer wieder zurückkommen.

Die Frage nach dem Verschwinden des Menschen wurde in den Literaturen, aber auch in Philosophie und Kulturwissenschaften immer wieder neu gestellt. Am vielleicht lustvollsten hat dies Michel Foucault in seinem Buch Les mots et les choses von 1966 getan, und zwar erstaunlicherweise wiederum im letzten Abschnitt seines Bandes, der übrigens mit einem Verweis auf Jorge Luis Borges und damit auf die Literaturen der Welt begann. Ich möchte Ihnen diese Passage gerne nachfolgend präsentieren, da sie uns etwas vom Pathos jener Kernfragen vermittelt, welche in den sechziger Jahren aufgeworfen und höchst kontrovers diskutiert wurden:

Eines ist auf jeden Fall gewiß: der Mensch ist nicht das älteste und auch nicht das konstanteste Problem, das sich dem menschlichen Wissen gestellt hat. Wenn man eine ziemlich kurze Zeitspanne und einen begrenzten geographischen Ausschnitt herausnimmt - die europäische Kultur seit dem sechzehnten Jahrhundert -, kann man sicher sein, dass der Mensch eine junge Erfindung ist. Nicht um ihn und um seine Geheimnisse herum hat das Wissen lange Zeit im Dunkeln getappt. Tatsächlich hat unter den Veränderungen, die das Wissen von den Dingen und ihrer Ordnung, das Wissen der Identitäten, der Unterschiede, der Merkmale, der Äquivalenzen, der Wörter berührt haben - kurz inmitten all der Episoden der tiefen Geschichte des Gleichen -, eine einzige, die vor anderthalb Jahrhunderten begonnen hat und sich vielleicht jetzt abschließt, die Gestalt des Menschen erscheinen lassen. Es ist nicht die Befreiung von einer alten Unruhe, der Übergang einer Jahrtausende alten Sorge zu einem lichtvollen Bewußtsein, das Erreichen der Objektivität durch das, was lange Zeit in Glaubensvorstellungen und in Philosophien gefangen war: es war die Wirkung einer Veränderung in den fundamentalen Dispositionen des Wissens. Der Mensch ist eine Erfindung, 
deren junges Datum die Archäologie unseres Denkens ganz offen zeigt. Vielleicht auch das baldige Ende. Wenn diese Dispositionen verschwänden, so wie sie erschienen sind, wenn durch irgendein Ereignis, dessen Möglichkeit wir höchstens vorausahnen können, aber dessen Form oder Verheißung wir im Augenblick noch nicht kennen, diese Dispositionen ins Wanken gerieten, wie an der Grenze des 18. Jahrhunderts die Grundlage des klassischen Denkens es tat, dann kann man sehr wohl wetten, dass der Mensch verschwindet wie am Meeresufer ein Gesicht im Sand. ${ }^{4}$

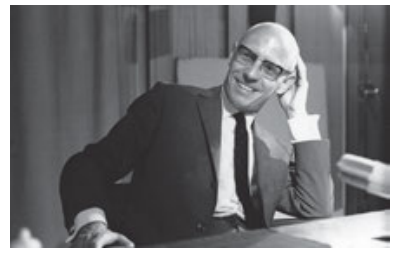

Abb. 90: Michel Foucault (Poitiers, 1926 - Paris, 1984).

Nach der Lektüre dieser Passage verstehen wir vielleicht besser, warum die Barthes'sche Bezeichnung als „sanfte Apokalypse“ nicht ungeeignet ist, weniger eine scharfe epistemologische Veränderung oder Bruchlinie als vielmehr eine sanfte Veränderung hinsichtlich der so oft beschworenen Sensibilitäten zu beschreiben, welche seit dem Ausgang der fünfziger Jahre immer stärker zu beobachten ist. Eine Apokalypse wäre das Verschwinden der Menschheit in toto sehr wohl. Sanft ist sie auch im abschließenden Bilde des Menschen im Sand am Meeresufer schon deshalb, weil hier keine großen Revolutionen, keine Brüche, keine Explosionen notwendig wären, um einer aktuellen Situation, um der Existenz des Menschen, der Existenz der Literatur, am Ende der Existenz eines Buches ein Ende zu bereiten. Es genügte ein einfaches Auswischen.

Es gibt eine zumindest vielen französischen Denkern eigene gemeinsame Sensibilität, die mit der Thematik und Metaphorik der Apokalypse verbunden ist. Daraus ergab sich eine vor allem im Bereich der Philosophie angesiedelte Debatte, die sich zwischen Frankreich und Deutschland, zwischen Paris und Frankfurt angesiedelt hat und die etwa von Manfred Frank in seine Sichtweise der Dinge grundlegend einbezogen wurde. ${ }^{5}$ Es sind also epistemologische und diskursive Strukturen, die - so können wir Foucaults Passage entnehmen - unsere eigene Existenz und unser Selbstverständnis als Menschen bestimmen, begründen und auch wieder aufkündigen. Sie stehen in Opposition oder zumindest in Konkurrenz zu anderen epistemologischen und diskursiven Systemen, die entweder in unserem Raum vor unserer Zeit oder auch in unserer Zeit außerhalb unseres

4 Foucault, Michel: Die Ordnung der Dinge. Frankfurt a.M.: Suhrkamp 1974, S. 462 f.

5 Vgl. Frank, Manfred: Was ist Neostrukturalismus? Frankfurt am Main: Suhrkamp 1983. 
Raumes vorhanden waren oder sind. Dies schließt eine Sichtweise mit ein, welche nicht mehr nur den Menschen, das Subjekt, die Literatur oder was auch immer aus dem Zentrum nimmt, sondern überhaupt auf zentrierte Figuren - und seien es jene des Eurozentrismus - verzichtet oder, sagen wir es vorsichtiger, verzichten $\mathrm{zu}$ können glaubt. Sie merken: Wir befinden uns an dieser Stelle plötzlich in einer ganz anderen geistes- und kulturgeschichtlichen Konstellation, welche im Verlauf der sechziger Jahre keineswegs nur in Europa der abendländischen Subjektphilosophie - und mit ihr natürlich auch der Philosophie Jean-Paul Sartres erfolgreich ein anderes Modell an die Seite stellte und den führenden Rang streitig machte. Dass dies - ich denke zu Unrecht - von einigen damaligen Zeitgenossen so ausgelegt wurde, als zielte dies alles allein auf Sartre und die als progressiv erachteten Vorstellungen eines (linken) politischen Engagements, sei zumindest erwähnt, um auf die Missverständnisse in damaligen Kommentaren, in welchen Foucault als „Totengräber der kritischen Linken“ bezeichnet wurde, hinzuweisen. Diskutieren möchte ich dies in unserer Vorlesung jedoch nicht mehr.

Wir werden uns an späterer Stelle noch länger mit Michel Foucault beschäftigen. Bereits jetzt sei festgehalten, dass seine Schriften ein Element in die „archäologische“ Untersuchung von Denksystemen, der Beziehungen zwischen den Worten und den Dingen einführten, das - wie wir noch sehen werden - für das Denken im Zeichen der Postmoderne von zentraler Bedeutung sein sollte. Es ging um die eigene Endlichkeit, die eigene Relativität, aber auch den eigenen Untergang und vielleicht sogar die eigene Lust daran. Apocalypse now - der Titel dieses berühmten Vietnam-Films von Francis Ford Coppola aus dem Jahr 1979 steht für eine grundlegende Veränderung, die sich diesseits und jenseits der sanften Apokalypse und damit diesseits und jenseits des Scharniers von Moderne und Postmoderne ansiedelt.

Diese Forderung nach einer Apokalypse - und zwar bitte gleich hier und sofort - mag uns an die Gruppe The Doors erinnern mit ihrem 1967 veröffentlichten Song und Jim Morrisons berühmtem Schrei „We want the world and we want it now“. Die Reiter der Apokalypse finden sich ebenfalls rasch in anderen Bereichen des Geisteslebens der damaligen Jahre, und davon waren glücklicherweise die Geisteswissenschaften nicht ausgeschlossen.

So hat der deutsche, in die USA ausgewanderte Romanist Hans Ulrich Gumbrecht - wir schreiben das Jahr 1985 (und daran sehen sie, dass nicht alles in den Geisteswissenschaften sich mit elektronischer Digitalgeschwindigkeit vollzieht) in einem kurzen Essay mit dem sprechenden Titel Posthistoire Now den Finger in eine Wunde gelegt, die wir bei allen bereits angeführten Autoren beobachten konnten. Denn die schlichte These seiner Reflexionen betonte die Erkenntnis, dass das Zeitbewusstsein, das Geschichtsbewusstsein, längst ins Wanken geraten war und einem neuen Zeitempfinden den Gestaltungsraum überlassen hatte. In 
seinen an die Erkenntnisse Michel Foucaults aus den sechziger Jahren angelehnten Überlegungen ging es weniger um die Erfindung des Menschen als um die Erfindung der Geschichte. Aber auch die hatte es fürwahr in sich.

Denn diese Geschichte der Moderne beginnt im letzten Drittel des 18. Jahrhunderts mit einem zukunftsorientierten und zukunftsoffenen, nicht mehr in einem zyklischen Geschichtsverständnis verfangenen Geschichtsbegriff, in welchem zugleich der Kollektivsingular der Geschichte entsteht ${ }^{6}$ und die Geschichte in alle Bereiche des Denkens - in Anthropologie, Biologie, Geologie oder Zoologie - eindringt. ${ }^{7}$ Diese fundamentalen Veränderungen treten in den Forschungen Foucaults, in seiner Archäologie kulturell bedingter Gewissheiten, deutlich hervor.

Alle Phänomene und Erscheinungen der organischen wie anorganischen Welt - mithin ebenso die Säugetiere, die Reptilien oder die Quallen, aber auch die Sedimentgesteine, die Ammoniten oder die Vulkane - werden vom Zeitpfeil durchdrungen und haben fortan eine Geschichte. Die Entwicklungsgeschichte geologischer wie geomorphologischer Formen wie etwa der Vergletscherungsformen in Europa treten ebenso in das Bewusstsein des Menschen wie seine eigene Entwicklungsgeschichte als Spezies oder die epistemischen Grundlagen, auf denen sich sein Denken vorwärtstastet. Sie stehen nicht länger in einem Tableau nebeneinander, sondern formen sich zu einer Geschichte, in welche sich alles einzuordnen beginnt und alles einzuordnen hat.

Auf den unterschiedlichsten Bereichen vollzieht sich so seit der Spätaufklärung der Prozess der Moderne, der uns als ein Erbe des ausgehenden 18. Jahrhunderts anvertraut ist - wie uns unsere Vorlesungen zur Aufklärung zwischen zwei Welten zeigen - und der sich im Horizont veränderter Sensibilitäten in der zweiten Hälfte des 20. Jahrhunderts im Zeichen der Postmoderne verändert. Die Gewissheiten des Strukturalismus lösten sich im Zeichen des anstürmenden Poststrukturalismus - und die großen Protagonisten dieser Entwicklung wie Michel Foucault oder Roland Barthes gingen zunächst einmal vom Strukturalismus aus ebenso auf wie die Gewissheiten der Moderne unter dem Ansturm postmoderner Denkfiguren, die Öffnungen und Hohlräume erkannten, in welche das Denken im Zeichen der Postmoderne einzudringen vermochte. Dies waren grundlegende Umbesetzungen, die wir im Bereich der Philosophie oder der Kulturwissenschaf-

6 Vgl. hierzu die wichtigen historiographischen Untersuchungen von Koselleck, Reinhart: Vergangene Zukunft. Zur Semantik geschichtlicher Zeiten. Frankfurt am Main: Suhrkamp 1979.

7 Vgl. neben den Arbeiten Foucaults auch Lepenies, Wolf: Das Ende der Naturgeschichte. Wandel kultureller Selbstverständlichkeiten in den Wissenschaften des 18. und 19. Jahrhunderts. Frankfurt am Main: Suhrkamp 1978. 
ten in der zweiten Hälfte des 20. Jahrhunderts sich vollziehen sehen, die aber im Bereich der Literaturen der Welt sich bereits Jahrzehnte früher vollzogen, wie wir im Anschluss anhand einer Reihe literarischer Beispiele erkennen werden.

Doch schauen wir noch einmal in die Mitte der achtziger Jahre. $\mathrm{Zu}$ einem zeitlich bereits weit fortgeschrittenen Zeitpunkt ging Hans Ulrich Gumbrecht um bei einem Vertreter unseres Fachs Romanistik zu bleiben - zunächst von der Frage eines Zusammenhangs zwischen Geschichtsschreibung und kollektiver Identität aus und fragte sich, ob Identitätsvergewisserung heute noch erfolgreich mit Hilfe von Geschichtsschreibung bewältigt und angestrebt werden könne. Die Idee der „Posthistoire“, so Gumbrecht, finde sich schon früh, etwa auch bei Hegel oder Marx, erschiene nun aber in einem anderen Licht aus der Gewissheit heraus, dass die großen Versprechen der Aufklärung nicht mehr einzuhalten sein würden. Letztere erwiesen sich mittlerweile als unerreichbare Illusionen, ja im Zeichen von Max Horkheimers und Theodor W. Adornos Dialektik der Aufklärung als gefährliche Traumbilder (wie wir hinzufügen könnten).

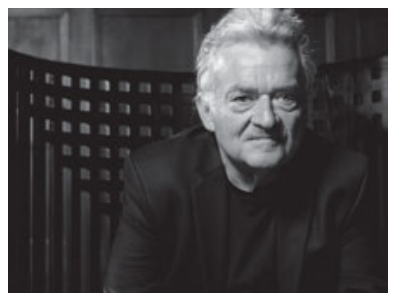

Abb. 91: Hans Ulrich Gumbrecht (Würzburg, 1948).

Die Gegenwart, so Gumbrecht in Rückgriff auf Koselleck, erscheine als die Zukunft der Vergangenheit. Die Dimension der Perfektibilität verliere an Bedeutung, so könnten wir interpretieren. Es habe eine Wiedergeburt der Aufklärung gegeben, aber eben als Fehlgeburt. In direkter Auseinandersetzung mit Jürgen Habermas' Rede vom „unvollendeten Projekt der Moderne“8 versicherte Gumbrecht rundweg, die Aufklärung halte heute keine Lösungspotentiale mehr für die Zukunft bereit. Damit aber könne der Zukunftshorizont zur Bedrohung geraten ein Hinweis darauf, wie sich die sanfte Apokalypse unserer französischen Meister-Denker auch denken ließe - und ich betone: denken ließe. Ich bin jedenfalls der Ansicht, dass selbst Gumbrecht heute nicht mehr seiner eigenen Deutung vorbehaltlos zustimmen würde. Aber sein nachfolgendes Zitat macht sehr wohl deutlich, worum es ihm - und so vielen anderen - in den achtziger Jahren ging.

8 Vgl. Habermas, Jürgen: Die Moderne - ein unvollendetes Projekt (1980). In (ders.): Kleine Politische Schriften (I - IV). Frankfurt am Main: Suhrkamp 1981, S. 444-466. 
Diese Überzeugungen sind wichtig, um die Umbesetzungen zu verstehen, die sich zwischen den historischen Avantgarden und den verschiedenen Ausprägungsformen eines Schreibens im Zeichen der Postmoderne entwickelten. Dass sich unter dem Ansturm postmoderner Geschichtsauffassungen auch der ModerneBegriff zunehmend präzisierte und ausdifferenzierte, mögen unter vielen anderen Beispielen Jürgen Habermas' Überlegungen zum philosophischen Diskurs der Moderne just aus demselben Jahr 1985 belegen. ${ }^{9}$

Laut Gumbrecht sei es das Ziel, die Geschichte nun anders zu denken und einen (nicht näher definierten) geschichtlichen Begriff von Geschichte zu entwickeln. Wenn wir aber Geschichte anders denken sollen, wie sollen wir sie dann denken? Schauen wir mal, wie zwanzig Jahre nach Michel Foucaults Veröffentlichung seiner Passage zum Verlöschen oder Tode des Menschen die Antwort darauf ausfällt, wie denn Geschichte nunmehr zu denken sei. Es überrascht freilich nicht, dass die Antwort darauf im Modus des „vielleicht“ ausfällt:

Was wären Grundelemente eines neuen Begriffs von Geschichte? Vielleicht die Ersetzung des einen Telos durch viele Nahziele? vielleicht die Substitution des Begriffes von der „Perfektibilität“durch einen Begriff von der Erhaltung des Menschen; vielleicht die Ablösung des (zur Vervollkommnung aufgerufenen) geistigen durch den (um seine Erhaltung besorgten) physischen Menschen. ${ }^{10}$

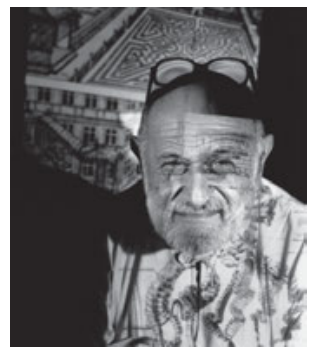

Abb. 92: Vilém Flusser (Prag, 1920 - Bor, Tschechien, 1991).

Sie sehen: Die Antworten auf diese Fragen sind immer nur vorläufig und halten sich von ihrer Programmatik her deutlich in Grenzen. In den Literaturen der Welt waren die Antworten auf derartige Fragen wesentlich früher präsent und gingen in wesentlich stärkerem Maße in konkrete fiktionale Strukturen ein. Die grund-

9 Vgl. Habermas, Jürgen: Der philosophische Diskurs der Moderne. Zwölf Vorlesungen. Frankfurt am Main: Surkamp 1985.

10 Gumbrecht, Hans Ulrich: Posthistoire Now. In (ders. / Link-Heer, Ursula. Hg.): Epochenschwellen und Epochenstrukturen im Diskurs der Literatur- und Sprachhistorie. Frankfurt a.M. 1985, S. 34-52, hier S. 48. 
legende Geste ist nicht die einer konstruktiven Antwort auf Herausforderungen, sondern die einer Dekonstruktion bestehender Begriffe, welche die Moderne ins Spiel gebracht hat. $\mathrm{Zu}$ diesen von der Moderne ,erfundenen' Begrifflichkeiten zählt etwa der aufklärerische, von Rousseau in Umlauf gesetzte Neologismus der „perfectibilité“, der aus der Feder des Genfer Bürgers stammend eine so folgenreiche kulturtheoretische wie philosophische Karriere hinzulegen wusste.

Um die Umbesetzungen und das Umsteuern dieser Jahre besser zu verstehen, ist auch jener Hinweis hilfreich, den uns der aus einer jüdischen Gelehrten- und Akademikerfamilie stammende und aus Prag nach Brasilien emigrierte Philosoph, Medientheoretiker und Kommunikationsforscher Vilém Flusser in deutlicher Anspielung auf François Lyotards Thesen von den großen Meistererzählungen einerseits und auf die Posthistoire-Diskussion andererseits geben kann. Dieser Hinweis gilt selbstverständlich auch für diese Vorlesung selbst und damit für die Geschichte, die ich in ihr über mehrere lange Sitzungen hinweg erzählen will.

Denn das Geschichtenerzählen ist ein Bereich, der seit den beiden sehr unterschiedlichen Historikerstandpunkten eines Golo Mann oder eines Hayden White von der Geschichtswissenschaft immer wieder anders problematisiert wurde. In letzter Instanz trugen diese oft mit großer Vehemenz geführten Debatten höchstens dazu bei, die Lust am Narrativ, die Lust am Geschichtenerzählen und am Geschichtenhören nur noch zu steigern. Das narrative Element ist zweifellos der Treibstoff, mit dessen Hilfe unsere Diskurse in Bewegung bleiben und in der Vervielfachung des Sinnes und der Sinne funktionieren. Ich bin, so darf ich hinzufügen, zuversichtlich, dass mir in dieser Vorlesung der Treibstoff nicht ausgehen wird.

Sehen wir uns aber nun eine kurze Passage aus Flussers 1991 - der Philosoph verstarb im Übrigen im selben Jahr am 27. November - verfasstem Text Vom Ende der Geschichte näher an:

Posthistoriker, Leute, die vom Ende der Geschichte erzählen, sind notwendigerweise Geschichtenerzähler. Wenn sie vom Ende der Geschichte erzählen, machen sie Geschichte. Es sieht so aus, als seien sie in ein geradezu sophistisches Paradox verstrickt, so ähnlich wie jemand, der vom Ende der Philosophie spricht und mit dieser philosophischen Aussage die Philosophie vorantreibt. Dennoch ist es nicht widersprüchlich, vom Ende der Geschichte zu sprechen. ${ }^{11}$

11 Flusser, Vilém: Vom Ende der Geschichte, In: Nachgeschichte. Eine korrigierte Geschichtsschreibung. Bensheim: Fischer Wissenschaft 1993, S. 282-290, hier S. $282 \mathrm{f}$. 
Wir sind an dieser Stelle unserer Vorlesung bereits ein erstes Mal in eine Vielzahl an Themen, Leitmotiven und Obertönen der Diskussionen und Debatten um Moderne und Postmoderne eingetaucht und werden uns mit diesen herausfordernden Fragestellungen intensiv vom Standpunkt der Literaturen der Welt auseinandersetzen. Ich möchte diese Auseinandersetzung also nicht - wie dies in Deutschland vorrangig geschah - auf dem Feld der Philosophie führen, obwohl die Philosophie, wie Sie schon gemerkt haben, ein gewichtiges Wörtchen in dieser Debatte mitzureden hat. Mit anderen Worten: Ich möchte der Literatur nicht den Rang einer kleinen Schwester geben, die von ihrer großen Schwester namens Philosophie gegängelt oder an die Hand genommen wird und nun unter Aufsicht jene Spiele spielt, die die große Schwester erlaubt und vielleicht selbst - natürlich auf höherem Niveau - zu spielen gewillt ist. Dies entspräche nicht meiner Sichtweise von Funktion und Bedeutung der Literaturen der Welt. Denn diese Literaturen sind jene Erprobungsräume, in denen ein Denken erstmals getestet und experimentell erprobt wird, bevor disziplinierte Wissensbereiche sich dieser dann erfolgreichen Experimentalanordnungen bedienen und sie ,disziplinieren".

Der Spielplatz der Literaturen der Welt ist weitaus größer und weniger festgelegt als jener der Philosophie - und insbesondere jener der akademischen abendländischen Philosophie. Ich bin daher der Ansicht, dass bisweilen eher die vermeintliche große Schwester Philosophie versucht hat, den Spielen der Literatur und anderer Künste Regeln zu entnehmen, die es auf ihr eigenes Spielfeld zu übertragen galt. Dabei könnte es sein, dass sie nicht nur bestimmte Regeln abgeleitet, sondern auch nicht wenige von ihnen übernommen hat, welche ihren Daseinsgrund veränderten (oder zumindest verändern müssten).

Denn was Roland Barthes am Übergang zu einer postmodernen Denkweise von der Literatur sagen konnte, könnte vielleicht auch für die Philosophie gelten: Sie wird heute nicht mehr (so) bewacht. Dies gilt freilich für die akademische Philosophie in Deutschland nicht, hat diese es doch erfolgreich geschafft, die interkulturelle Philosophie in ihrem Einflussbereich auf ein Minimum zu reduzieren und von deutschen Lehrstühlen weitestgehend zu verdrängen. Indes wäre auch in diesem Kontext einmal mehr Friedrich Nietzsche als Ahnherr anzuführen: ein Ahnherr freilich, der zwischen Philologie, Philosophie und Literatur steht und damit bestens dafür geeignet erscheint, die Grenzen zwischen diesen einst sorgsam abgeschirmten Bereichen zu durchbrechen.

Sie verstehen: Ich möchte, wie es auch meine Aufgabe ist, die Problematik von Moderne und Postmoderne im Kontext der Beziehungen zur Avantgarde - und damit im Kontext der literarischen Entwicklungen des 20. wie des beginnenden 21. Jahrhunderts - anhand von überprüfbaren Geschichten erzählen! Sie haben ihren Schwerpunkt im Bereich der Literatur und selbstverständlich möchte ich 
sie textanalytisch präsentieren, also wie stets und mit Hilfe eines Close Reading möglichst nahe an den literarischen Texten. Deshalb werde ich auch mit einem Schriftsteller und nicht mit einem Philosophen in diese neue Phase unserer Vorlesung eintreten, auch wenn wir längst durch ein philosophisch-literarisches Übergangstor in jenen Bereich vorgestoßen sind, der im Zeichen einer sanften Apokalypse und im Scharnierbereich zwischen Moderne und Postmoderne steht. Dass wir dabei zugleich mit einem Autor einsetzen, der aus Lateinamerika stammt und einen wesentlich komplexeren inter- und transkulturellen Hintergrund in sein Schreiben einbringt, ist aus Sicht des aktuellen Systems der Literaturen der Welt gewiss von großem Vorteil. ${ }^{12}$

Doch versuchen wir, unmittelbar vor unserer Beschäftigung mit dem Argentinier Jorge Luis Borges noch eine wichtige Verbindungslinie zu ziehen. Unser kleiner Durchgang umfasste bisher die im Zeichen der aufkommenden Postmoderne etwa von Michel Foucault vorgetragene Rede vom Ende des Menschen mitsamt der nicht weniger wichtigen und prominent von Roland Barthes vertretenen Rede vom Tode des Autors. Diese beiden Aspekte müssen wir mit jener Rede in Einklang bringen, welche soeben von Hans Ulrich Gumbrecht im Modus der Posthistoire mit dem Ziel einer Erhaltung des Menschen vorgetragen wurde. Erstaunlicherweise führt uns die Verbindung dieser drei Diskurse ein letztes Mal zum Anfang unserer gesamten Vorlesung und zu ihrem allerersten Zitat von Maurice Blanchot zurück.

Denn in Le livre á venir ging der französische Literaturkritiker bereits auf jenen Schriftsteller ein, der Jahrzehnte später als der geistige Vater der Postmoderne weltweit akklamiert werden sollte. Es war keineswegs ein Zufall, dass Jorge Luis Borges seine literarische Karriere im Zeichen der historischen Avantgarden begonnen hatte. Vielmehr liegt dieses Faktum ganz in der Logik unserer Vorlesung und völlig auf der Linie jener narrativen Struktur, welche sie quer durch das vergangene Jahrhundert verfolgt.

Blanchots Lektüre von Jorge Luis Borges legte einige der grundlegenden Themen des argentinischen Schriftstellers frei, die im weiteren Verlauf des Jahrhunderts - ganz im Sinne dieses Livre à venir - zu den zentralen Thematiken der Postmoderne avancieren wollten. Denn Blanchot begriff: „Aber wenn die Welt ein Buch ist, dann ist jedes Buch die Welt, und aus dieser unschuldigen Tautologie

12 Vgl. Ette, Ottmar: WeltFraktale. Wege durch die Literaturen der Welt. Stuttgart: J.B. Metzler Verlag 2017; sowie (ders.): Die Literaturen der Welt. Transkulturelle Bedingungen und polylogische Herausforderungen eines prospektiven Konzepts. In: Lamping, Dieter / Tihanov, Galin (Hg.): Vergleichende Weltliteraturen / Comparative World Literatures. DFG-Symposion 2018. Unter Mitwirkung von Mathias Bormuth. Stuttgart: J.B. Metzler - Springer 2019, S. 115-130. 
ergeben sich Konsequenzen, die einem das Fürchten lehren. “13 Ich möchte Ihnen im Folgenden ganz bestimmt nicht das Fürchten lehren, aber Sie doch einführen in die literarisch wie philosophisch so reichen und vielfältigen Folgen dieser auf den ersten Blick so harmlosen Tautologie. Denn sie impliziert auch die grundlegende Infragestellung der nur auf den ersten Blick so scharfen Trennung zwischen Urbild und Abbild, Original und Fälschung, Realität und ... Realität.

Im Mittelpunkt der Literatur, so Blanchot, stehe nicht das Individuum, nicht der Mensch, sondern die Literatur, mithin das Buch; und im Buch wiederholen sich alle Bücher, die sich wechselseitig spiegeln und abbilden, ad infinitum. Die originäre Stimme eines Autors ist hier nicht länger auszumachen. Sie bemerken, dass auf diese Weise die Bedeutung des großen Autors, des bestimmenden Schöpfer-Subjekts, ganz erheblich schrumpft und dies so lange tut, bis es keine Rolle mehr spielt und man vom Tod des Autors sprechen könnte. Zugleich aber beginnt der Mensch, beginnt das Individuum, im Kreuzungspunkt dieser Texte oder Gewebe - denn Text meint letztlich ganz konkret das Gewobene - eine neue Gestalt anzunehmen, ein neues Leben zu entfalten. Die Erhaltung des Menschen steht im Zeichen all der Texte, die dieses Individuum queren und es nähren. So überrascht es nicht, wenn Maurice Blanchot gerade von jenem Werk beeindruckt ist, dem als Grundgedanke eine „absurdité mémorable“ zugrunde liege, jenem Buch über den im Bachtin'schen Sinne Ausgangspunkt des modernen europäischen Romans. Ich spreche von Miguel de Cervantes' Don Quijote und von Jorge Luis Borges' Pierre Menard, autor del Quijote. Denn nicht umsonst stehen bereits im Titel dieser borgesianischen Erzählung der Begriff des Autors und ein individueller Name.

Lassen Sie mich aber abschließend zu Maurice Blanchots Band aus dem Jahre 1959 sagen, dass es aus meiner Sicht ein viel zu wenig beachtetes und beobachtetes Unterfangen darstellt, neue Tendenzen in der Literatur festzuhalten und mit überaus sensiblem Gespür richtungsweisende Neuentwicklungen aufzuspüren und - wo möglich - zu prognostizieren. Dieser kleine Band ist ein Schatzkästlein. Sie finden auf seinen Seiten wesentliche Grundgedanken der Ästhetiken der sich zumindest bis zum Jahrhundertende anschließenden Jahrzehnte vor: vom Schwund der Literatur (der an die „exhaustion“ von John Barth erinnert) bis hin zum „Tod des letzten Schriftstellers“. Dieser Tod weist in manchem schon voraus auf die programmatischen Essays von Roland Barthes (1967) über La mort de l'auteur oder von Michel Foucault (1969) zur Frage Qu'est-ce qu'un auteur? Sie finden in Blanchots Band die Problematisierung des textexternen Referenten ebenso wie die Infragestellung einer linearen Literaturentwicklung und ihrer

13 Blanchot, Maurice: Le livre à venir, S. 131. 
Institutionen - und wie problematisch es ist, sich einer linearen Geschichte wie ihrer „Großen Erzählung“ einfach hinzugeben.

Ich möchte freilich, dass Sie sich in dieser Welt von Spiegeln, die sich wechselseitig spiegeln, in dieser gleichsam erlesenen Spiegelgalerie nicht mit all ihren Lektüren verlieren, sondern auch weiterhin den Durchblick behalten. Denn wir wollen verstehen, wie genau die möglichen Wege von den historischen Avantgarden durch ein langes 20. Jahrhundert bis in die Gegenwartsliteraturen, bis hin ins „extrême contemporain“ der ersten Jahrzehnte des 21. Jahrhunderts verliefen. Wir wollen begreifen, wie sich eine Literatur, die sich als modern verstand und noch nicht wissen konnte, dass sie einmal postmodern gedeutet werden könnte. Eine Literatur, die den Raum der Moderne zu gestalten versuchte und noch nicht ahnen konnte, dass ihr ein Raum zugeschrieben wurde, in dessen Flächen und Nischen andere Gottheiten stehen sollten. Wir wollen also nachvollziehen, wie eine Literatur entstand, die sich aus einer globalen weltumspannenden Sichtweise von Weltliteratur in jene der Literaturen der Welt verwandelte, welche heute die literarischen Entwicklungen in einem viellogischen, polylogischen System prägen.

Wie auch immer der Raum und die Räumlichkeiten gestaltet sein mögen, welche die Postmoderne charakterisieren; ob wir diese Räumlichkeiten der Postmoderne noch zu denen der Moderne hinzurechnen oder ihnen einen eigenen, eigenständigen oder gar unabhängigen Status zubilligen: Eine der Gottheiten, die in diesen Räumen schon früh aufgestellt wurde und lange Zeit in ihnen wachte, stammte aus Lateinamerika. Und mit dieser Figur, die in Umberto Ecos Il nome della rosa als der Wächter über die Bibliothek namens Jorge de Burgos mit Argusaugen auf seine Klosterbrüder sieht, wollen wir uns nun beschäftigen. 